Gut and Liver, Vol. 12, No. 6, November 2018, pp. 648-654

\title{
An Economic Modeling Study of Helicobacter pylori Eradication: Comparison of Dual Priming Oligonucleotide-Based Multiplex Polymerase Chain Reaction and Empirical Treatment
}

\author{
Tae-Geun Gweon, Joon Sung Kim, and Byung-Wook Kim
}

Division of Gastroenterology, Department of Internal Medicine, Incheon St. Mary's Hospital, College of Medicine, The Catholic University of Korea, Seoul, Korea

Background/Aims: Dual priming oligonucleotide-based multiplex polymerase chain reaction (DPO-based PCR) can detect the presence of clarithromycin resistance without culture. The aim of this study was to investigate the costeffectiveness of DPO-based PCR for Helicobacter pylori eradication. Methods: From 2015 to 2016, medical records of patients who received $H$. pylori eradication therapy were analyzed. Patients were divided into two groups: tailored group patients who were treated based on DPO-based PCR and empirical group patients. Eradication rate and medical cost, including diagnostic tests, eradication regimens, and ${ }^{13} \mathrm{C}$-urea breath tests, were compared between the two groups. Cost for one successful eradication was calculated in each group. The expected cost of eradication for empirical treatment was investigated by varying the treatment duration and eradication rate. Results: A total of 527 patients were analyzed (tailored group 208, empirical group 319). The eradication success rate of the first-line therapy was higher in the tailored group compared to that in the empirical group (91.8\% vs $72.1 \%, p<0.01)$. The total medical cost for each group was $114.8 \pm 14.1$ U.S. dollars (USD) and $85.8 \pm 24.4$ USD, respectively $(p<0.01)$. The total medical costs for each ultimately successful eradication in the tailored group and in the empirical group were 120.0 USD and 92.4 USD, respectively. The economic modeling expected cost of a successful eradication after a 7- or 14-day empirical treatment was 93.8 to 111.4 USD and 126.3 to 149.9 USD, respectively. Conclusions: Based on economic modeling, the cost for a successful eradication using DPO-based PCR would be similar or superior to the expected cost of a successful eradication with a 14-day empirical treatment when the first-line eradication rate is $\leq 80 \%$. (Gut Liver 2018;12:648-654)

Key Words: Helicobacter pylori; Clarithromycin; Polymerase chain reaction; Cost

\section{INTRODUCTION}

Eradication rate of Helicobacter pylori has been declining for the past decades. ${ }^{1-4}$ Clarithromycin resistance has been reported as an essential factor for eradication failure. Clarithromycinbased triple therapy (TT), a combination of clarithromycin, amoxicillin and proton pump inhibitor (PPI), is recommended as first-line regimen in areas where clarithromycin resistance is less than $15 \% .^{5}$ However, clarithromycin resistance has been increasing in Asian countries including Korea for the past decades. ${ }^{6,7}$ Bismuth-based quadruple therapy (BQT), a combination of bismuth, PPI, metronidazole, and tetracycline, was generally adopted as second-line therapy because of its dose complexity and adverse events. ${ }^{8,9}$

Several treatment strategies have been introduced to improve eradication rates; first, development of alternative regimen, ${ }^{10-12}$ second, longer duration of therapy, ${ }^{10}$ and third, performing antimicrobial susceptibility test before treatment. ${ }^{13-16}$ Antimicrobial susceptibility test before $H$. pylori eradication therapy enables tailored therapy according to the results and showed better outcomes. ${ }^{14-16}$ However, culture-based antimicrobial susceptibility test is time consuming and inconvenient as a routine clinical practice due to its cost and low success rate. ${ }^{17}$

Recently, clarithromycin resistance test using dual priming oligonucleotide-based multiplex polymerase chain reaction (DPO-based PCR) has been introduced. DPO-based PCR can de-

Correspondence to: Byung-Wook Kim

Division of Gastroenterology, Department of Internal Medicine, Incheon St. Mary's Hospital, College of Medicine, The Catholic University of Korea, 56 Dongsu-ro, Bupyeong-gu, Incheon 21431, Korea

Tel: +82-32-280-5052, Fax: +82-32-280-5987, E-mail: gastro@catholic.ac.kr

Received on February 5, 2018. Revised on May 16, 2018. Accepted on June 18, 2018. Published online October 29, 2018

pISSN 1976-2283 eISSN 2005-1212 https://doi.org/10.5009/gnl18079

() This is an Open Access article distributed under the terms of the Creative Commons Attribution Non-Commercial License (http://creativecommons.org/licenses/by-nc/4.0) which permits unrestricted non-commercial use, distribution, and reproduction in any medium, provided the original work is properly cited. 
tect $H$. pylori itself and mutation of 23sRNA which is essential for clarithromycin resistance. ${ }^{18,19}$ It is convenient to use and the results are obtained within a short period. The results of DPObased PCR enable tailored eradication. ${ }^{20}$ However, the costeffectiveness of DPO-based PCR has not been investigated so far. The aim of this study was to evaluate the cost-effectiveness of DPO-based PCR for H. pylori eradication using an economic modeling analysis.

\section{MATERIALS AND METHODS}

\section{Study population}

The Institutional Review Boards (IRBs) of The Catholic University of Korea approved this study (IRB approval number: 0C17RESI0087). Medical records of patients' cohort who received eradication therapy for $H$. pylori between January 2015 and December 2016 in Incheon St. Mary's Hospital, Korea, were reviewed. All the study subjects underwent esophagogastroduodenoscopy (EGD) and the presence of $H$. pylori was confirmed by rapid urease test (RUT; Bio Helicobacter Test, Shinsung Pharmaceutical Co., Ltd, Suwon, Korea), histology such as Giemsa stain, or DPO-based PCR under physician's discretion. DPObased PCR was performed using Seeplex ${ }^{\circledR} H$. pylori-ClaR ACE Detection kit (Seegene Inc., Seoul, Korea). DPO-based PCR can detect A2142G and A2143G mutations of 23sRNA genes associated with clarithromycin resistance of $H$. pylori. Two pieces of gastric tissues were obtained for RUT and DPO-based PCR: one piece at antrum and corpus. For histology, four pieces of gastric mucosal biopsy were obtained: two pieces at antrum and corpus. Status of RUT was judged within 24 hours of sampling. H. pylori eradication was confirmed by ${ }^{13} \mathrm{C}$-urea breath test (UBT; Korea Otsuka Pharmaceutical Co., Ltd., Seoul, Korea) 4 to 6 weeks after eradication therapy. Inclusion criteria were as follows: (1) patients diagnosed with $H$. pylori; (2) patients who received eradication therapy; (3) patients confirmed of eradication 4 to 6 weeks after completion of treatment. Patients who received concomitant therapy or sequential therapy for clinical trials were excluded.

Patients were divided into two groups: (1) tailored group, those who underwent eradication therapy according to the results of DPO-based PCR; (2) empirical group, those who underwent eradication therapy without the results of DPO-based PCR. In the tailored group, TT was prescribed for the first-line eradication regimen in patients without clarithromycin resistance. For those who showed clarithromycin resistance, BQT or a combination of PPI, amoxicillin, metronidazole (PAM) was mainly prescribed as the first-line eradication regimen. For those who received TT as the first-line eradication regimen, the second-line eradication was conducted with BQT. For the second-line eradication therapy in patients with clarithromycin resistance, PAM was prescribed when BQT as the first-line therapy was failed. For those who received PAM as the first-line therapy, BQT was prescribed as the second-line therapy.

In the empirical group, TT was adopted as the first-line eradication regimen. BQT was followed as the second-line eradication regimen when TT was failed.

\section{Outcomes}

Total medical cost per patient was calculated in each group. The medical cost was sum of patient's payment and copayment of National Health Insurance. The cost of diagnosis for $H$. pylori infection, confirmation of $H$. pylori status after eradication therapy, and the cost of eradication regimen (cost of eradication regimen per dayxeradication period) were calculated. Cost for outpatient clinic registration and EGD were not included. Medical cost for RUT, histology, and DPO-based PCR was 9.3, 10.1, and 52.2 USD, respectively. Cost for TT, BQT, and PAM per day were 3.6, 2.7, and 1.9 USD, respectively. ${ }^{13} \mathrm{C}$-UBT costed 37.1 USD. Additional costs, cost of the second-line eradication and ${ }^{13} \mathrm{C}$-UBT, were calculated when the first-line therapy failed. The first-line eradication, the second-line eradication, and the ultimate eradication rate were investigated in each group. The results of DPO-based PCR were investigated in the tailored group.

Based on the medical cost and eradication rate, an economic model of empirical treatment for $H$. pylori was investigated by treatment duration and eradication rate of empirical treatment. ${ }^{21}$

\section{Statistics}

Total medical cost per patient and eradication rates were compared between the two groups. In comparing the two groups, we used student t-test for continuous variables. Chi-square test and Fisher exact test were used for comparison of categorical variables. $\mathrm{p}<0.05$ was considered significant statistically. Cost for one-successful eradication was calculated as follows.

Cost for one-successful eradication=total medical cost/number of patients eradicated.

In the economic model, cost for expected one-successful eradication for empirical treatment was calculated. Variables included in the economic model were as follows: the first-line eradication rate (50\%, 60\%, 70\%, 80\%); the ultimate eradication rate $(90 \%, 95 \%)$; eradication duration (7 days, 14 days). In the economic model, total cost per patient and expected cost for 1 successful eradication was calculated as the same manner as we calculated the medical cost in the present study. For example, assuming that 100 patients received empirical treatment for 7 days with the-first line eradication rate of 70\%, 70 patients finished eradication with 7-day TT and checked ${ }^{13} \mathrm{C}$-UBT once. Remaining 30 patients who failed the first-line eradication received eradication regimen twice (7-day TT and 7-day BQT) and tested ${ }^{13} \mathrm{C}$-UBT twice. In the economic model, cost for diagnostic test was set at 10 USD. Total medical cost in the economic model was as follows.

Total medical cost for 100 patients who received eradication regimen for 7 days: 70 (10 USD+3.6 USD $\times 7+37.1$ USD)+30 (10 
USD+3.6 USD ×7+2.7 USD ×7+37.1×2)=8,910 USD.

Assuming that the ultimate eradication rate was set at $90 \%$, cost per one-successful eradication was $99 \mathrm{USD}=8,910$ USD/100/0.9.

Break-even point was defined where the cost of empirical treatment of the economic modeling was equal to the tailored treatment of current study. All statistical analyses were performed using SAS software (SAS Institute, Cary, NC, USA).

\section{RESULTS}

\section{Patients' characteristics}

During this period, 2,269 patients were identified as $H$. pylori positive. Among them, 667 patients received eradication therapy. One hundred twenty-three patients who received concomitant therapy or sequential therapy were excluded. After exclusion of patients who were lost to follow up or took less than $80 \%$ drugs, 527 patients were included in the per-protocol analysis (Fig. 1).

The baseline characteristics of the two groups are shown in Table 1 . The mean age of the tailored group was higher than that of the empirical group $(\mathrm{p}=0.01)$. There were no significant differences in gender ratio, body mass index, or previous history of $H$. pylori eradication between the two groups. Previous eradication history was available in 140 of 527 patients (26.6\%).

\section{DPO-based PCR}

DPO-based PCR was performed in 462 patients (Fig. 1). Clar-

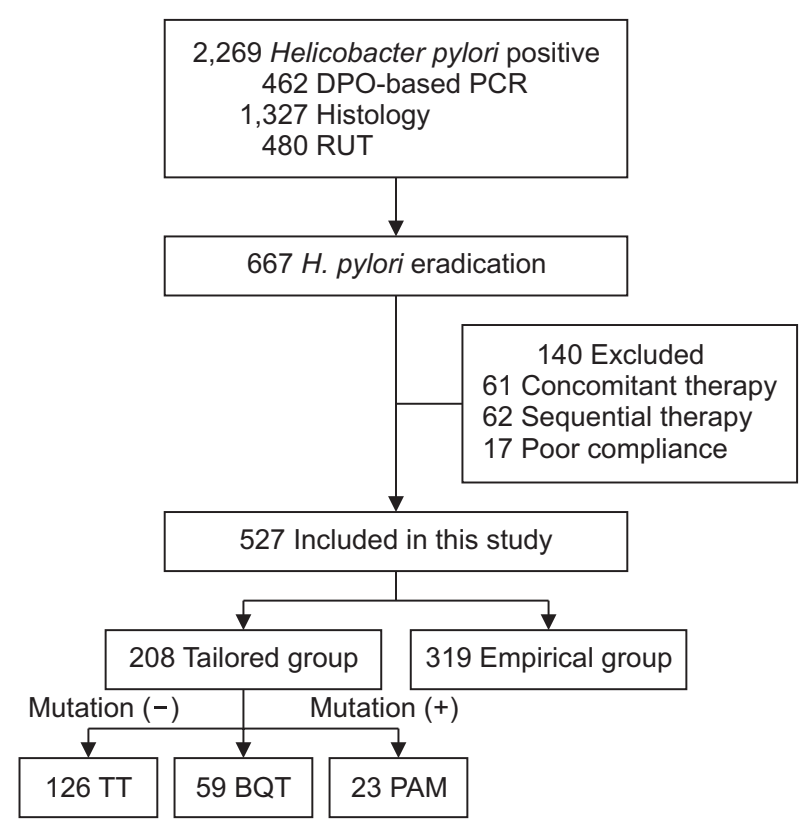

Fig. 1. Flow diagram of this study.

DPO-based PCR, dual priming oligonucleotide-based polymerase chain reaction; RUT, rapid urease test; TT, triple therapy (proton pump inhibitor [PPI], amoxicillin, clarithromycin); BQT, bismuth quadruple therapy; PAM, PPI, amoxicillin, metronidazole. ithromycin resistance by DPO-based PCR was found in 37.4\% (173/462).

\section{H. pylori eradication rate}

Table 2 shows the results of $H$. pylori eradication therapy. For the first-line eradication, 513 (97.5\%) and 14 (2.5\%) patients received eradication regimen for 7 days and 14 days, respectively. Eradication period was extended in some patients who had previous history of $H$. pylori eradication. In the tailored group, 126 patients (60.6\%) received TT in patients with clarithromycin resistance negative $H$. pylori. For 82 patients (39.4\%) who were infected with clarithromycin resistant $H$. pylori on DPO-based PCR, 59 patients (28.4\%) received BQT and 23 patients (11.0\%) received PAM as the first-line therapies. In the empirical group, all of the 319 patients (100\%) received TT as the first-line therapy. Eradication success rate of the first-line therapy was higher

Table 1. Baseline Characteristics of Patients Included in This Study

\begin{tabular}{lccc}
\hline \multicolumn{1}{c}{ Characteristics } & $\begin{array}{c}\text { Tailored } \\
\text { group }(\mathrm{n}=208)\end{array}$ & $\begin{array}{c}\text { Empirical } \\
\text { group }(\mathrm{n}=319)\end{array}$ & p-value \\
\hline Age, yr & $58.4 \pm 12.2$ & $56.2 \pm 10.5$ & 0.03 \\
Male sex & $138(66.3)$ & $191(59.9)$ & 0.13 \\
BMI, kg/m & $24.2 \pm 3.3$ & $24.1 \pm 3.3$ & 0.69 \\
Indication for endoscopy & & & $<0.01$ \\
$\quad$ Screening & $14(6.7)$ & $167(52.4)$ & \\
$\quad$ ESD, EMR & $102(49.0)$ & $53(16.6)$ & \\
$\quad$ Functional upper & $63(30.4)$ & $79(24.8)$ & \\
$\quad$ GI & & & \\
$\quad$ Bleeding, anemia & $29(13.9)$ & $20(6.2)$ & \\
Previous history of eradication* & $9 / 31(29.0)$ & $39 / 109(35.8)$ & 0.49 \\
\hline
\end{tabular}

Data are presented as mean \pm SD or number (\%).

BMI, body mass index; ESD, endoscopic submucosal dissection; EMR, endoscopic mucosal resection; GI, gastrointestinal.

*Missing data for Helicobacter pylori eradication: 177 tailored group and 210 empirical group.

Table 2. The Eradication Rates in Each Group

\begin{tabular}{lrcc}
\hline \multicolumn{1}{c}{ Eradication rate } & $\begin{array}{c}\text { Tailored } \\
\text { group }(\mathrm{n}=208)\end{array}$ & $\begin{array}{c}\text { Empirical } \\
\text { group }(\mathrm{n}=319)\end{array}$ & p-value \\
\hline First-line eradication rate & $191(91.8)$ & $230(72.1)$ & $<0.01$ \\
TT & $124 / 126(98.4)$ & $230(72.1)$ & \\
BQT & $50 / 59(84.7)$ & & \\
PAM & $17 / 23(73.9)$ & & \\
Second-line eradication rate & $8 / 9(88.9)$ & $66 / 75(88.0)$ & 0.36 \\
TT $\rightarrow$ BQT & $2 / 2(100.0)$ & $66 / 75(88.0)$ & \\
BQT $\rightarrow$ PAM & $2 / 3(66.7)$ & & \\
PAM $\rightarrow$ BQT & $4 / 4(100.0)$ & & \\
Ultimate eradication rate & $199(95.7)$ & $296(92.8)$ & 0.18 \\
\hline
\end{tabular}

Data are presented as number (\%).

TT, triple therapy; BQT, bismuth quadruple therapy; PAM, proton pump inhibitor (PPI), amoxicillin, metronidazole. 
in the tailored group compared to the empirical group (91.8\% vs $72.1 \%, \mathrm{p}<0.01)$. Among who failed in the first-line eradication, 22 patients refused second-line therapy. Eighty-four patients received the-second-line eradication and checked the status of $H$. pylori. The second-line eradication rate in the tailored group and the empirical group was $88.9 \%$ and $88.0 \%$, respectively $(p=0.36)$. Most of patients $(91.7 \%, 77 / 84)$ received second-line eradication therapy for 7 days. Eradication regimen was extended to 14 days for seven patients who were clarithromycin resistant

The ultimate eradication rate including second-line therapy did not show any difference between the two groups (tailored group 95.7\% vs empirical group 92.8\%, p=0.18). In the tailored group, TT and the ultimate eradication rate was $98.4 \%$ $(124 / 126)$ and 100\% (126/126), respectively, when DPO-based PCR showed clarithromycin sensitive. However, when DPObased PCR showed clarithromycin resistant, the eradication rates of BQT and PAM were 84.7\% and 73.9\%, respectively $(\mathrm{p}=0.25)$ and the ultimate eradication rate was $89.0 \%$ (73/82). Among the patients who failed in the first-line therapy, 47.1\% (8/17) in the tailored group and 15.7\% (14/89) in the empirical group refused to receive second-line therapy. Duration of drug administration

Table 3. Medical Costs for Each Group

\begin{tabular}{cccc}
\hline \multicolumn{1}{c}{ Costs (USD) } & $\begin{array}{c}\text { Tailored } \\
\text { group }(\mathrm{n}=208)\end{array}$ & $\begin{array}{c}\text { Empirical } \\
\text { group }(\mathrm{n}=319)\end{array}$ & p-value \\
\hline Total costs per patient & $114.8 \pm 14.1$ & $85.8 \pm 24.4$ & $<0.01$ \\
Diagnostic method & $52.2 \pm 0$ & $10.0 \pm 0.3$ & $<0.01$ \\
Eradication regimen & $23.9 \pm 7.7$ & $30.4 \pm 9.4$ & $<0.01$ \\
${ }^{13}$ C-UBT & $38.7 \pm 7.6$ & $45.3 \pm 15.7$ & $<0.01$ \\
Total costs for 1 successful & 120.0 & 92.4 & - \\
eradication & & & \\
Diagnostic method & 54.6 & 10.8 & \\
Eradication regimen & 24.9 & 32.8 & \\
${ }^{13}$ C-UBT & 40.4 & 48.9 & \\
\hline
\end{tabular}

Data are presented as mean \pm SD.

USD, United States dollars; UBT, urea breath test. in the tailored group was shorter compared to the empirical group (7.7 days vs 9.1 days, $\mathrm{p}<0.01$ ).

\section{Medical costs}

Medical costs of each group are presented in Table 3. Total medical cost per patient in the tailored group and in the empirical group was $114.8 \pm 14.1$ and $85.8 \pm 24.4$ USD, respectively $(\mathrm{p}<0.01)$. Cost for diagnostic method was higher in the tailored group (52.2 \pm 0 USD vs $10.0 \pm 0.3$ USD, $\mathrm{p}<0.01$ ). Cost for eradication regimen and ${ }^{13} \mathrm{C}-\mathrm{UBT}$ was higher in the empirical group $(23.9 \pm 7.7$ USD vs $30.4 \pm 9.4$ USD, $\mathrm{p}<0.01 ; 38.7 \pm 7.6$ USD vs $45.3 \pm 15.7$ USD, $\mathrm{p}<0.01$, respectively). Total medical costs for one-successful eradication in the tailored group and in the empirical group were 120.0 USD, and 92.4 USD, respectively.

\section{Economic modeling of empirical treatment}

Economic modeling of empirical treatment is shown in Fig. 2 and in Table 4. Cost of TT and BQT, analyzed in the economic modelling were as follows: 7-day TT, 25.2 USD;14-day TT, 50.4 USD; 7-day BQT, 18.9 USD; 14-day BQT, 37.8 USD. For example, assuming the first-line and the ultimate eradication rate

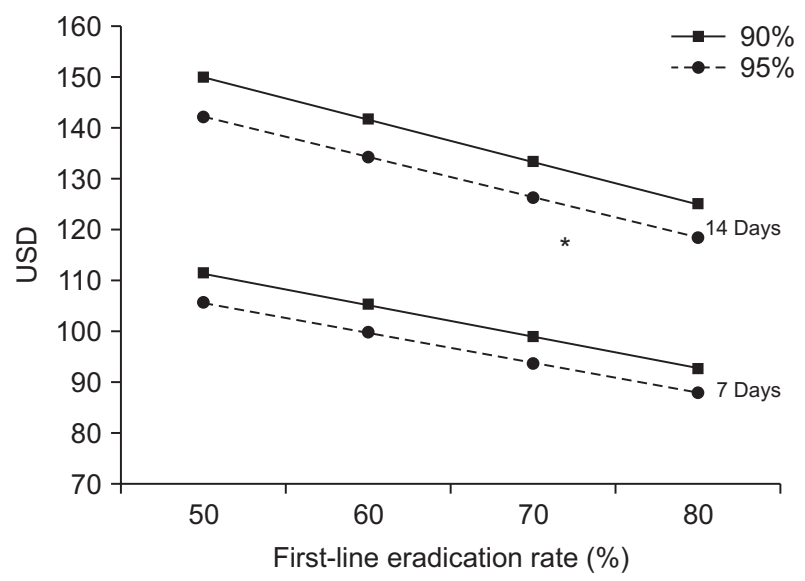

Fig. 2. Economic modeling of empirical treatment. UDS, United States dollars. *Indicates one-successful eradication for tailored group matching for empirical treatment.

Table 4. Economic Modelling of Empirical Treatment

\begin{tabular}{|c|c|c|c|c|c|c|c|c|}
\hline \multicolumn{9}{|l|}{ 7-Day treatment } \\
\hline First-line eradication success, $\%$ & 50 & 50 & 60 & 60 & 70 & 70 & 80 & 80 \\
\hline Ultimate eradication success, $\%$ & 90 & 95 & 90 & 95 & 90 & 95 & 90 & 95 \\
\hline Total cost per patient, USD & 100.3 & 100.3 & 94.7 & 94.7 & 89.1 & 89.1 & 83.5 & 83.5 \\
\hline Cost for 1 successful eradication, USD & 111.4 & 105.6 & 105.2 & 99.7 & 99.0 & 93.8 & 92.8 & 87.9 \\
\hline \multicolumn{9}{|l|}{ 14-Day treatment } \\
\hline First-line eradication success, $\%$ & 50 & 50 & 60 & 60 & 70 & 70 & 80 & 80 \\
\hline Ultimate eradication success, $\%$ & 90 & 95 & 90 & 95 & 90 & 95 & 90 & 95 \\
\hline Total cost per patient, USD & 135.0 & 135.0 & 127.5 & 127.5 & 120.0 & 120.0 & 112.5 & 112.5 \\
\hline Cost for 1 successful eradication, USD & 149.9 & 142.1 & 141.6 & 134.2 & 133.3 & 126.3 & 125.0 & 118.4 \\
\hline
\end{tabular}

USD, United States dollars. 
of 7-day empirical treatment of 50\% and 90\% respectively, total cost per patient and cost for one successful eradication would be 100.3 and 111.4 USD, respectively.

Expected cost for one-successful eradication of 7-day treatment and 14-day treatment were 87.9 to 111.4 USD and 118.4 to 149.9 USD, respectively. Break-even point was reached fulfilling the following conditions: (1) the first-line eradication rate: $80 \%$, (2) the ultimate eradication rate: $95 \%$, (3) duration of empirical treatment: 14 days. Cost of one-successful eradication for tailored group would be similar or superior compared to the expected cost for 14-day empirical treatment when the first-line eradication rate is $80 \%$ or less (Fig. 2). Given the eradication rate in the current study, tailored treatment using DPO-based PCR will be cost effective when the cost of DPO-based PCR is 24.6 USD, which is almost 30 USD cheaper than the current price.

\section{DISCUSSION}

This is the first investigation on the cost-effectiveness of clarithromycin resistance test using DPO-based PCR. In the current study, we compared the eradication rate and medical cost between those who underwent DPO-based PCR or not. Since the incidence of clarithromycin resistance was as high as 40\% in Korea, the eradication rate of the first-line therapy was higher in the tailored group. However, the ultimate eradication rate showed no difference between the tailored group and empirical group. Based on the economic model, considering the current medical cost and the eradication rate in Korea, cost for onesuccessful eradication using DPO-based PCR would be costeffective when the first-line eradication rate of 14-day empirical treatment is $80 \%$ or less.

DPO-based PCR results showed that clarithromycin resistance was $40 \%$, which was the highest compared to recent studies in Korea. ${ }^{7,20,22}$ It is plausible that this study included not only $H$. pylori eradication naïve patients but also patients who were exposed to previous $H$. pylori eradication therapy. Other possible cause is recent increase in clarithromycin resistance considering that clarithromycin resistance has been doubled in a short period (16.7\% during 2003-2005 vs 32.0\% during 2007-2009, $\mathrm{p}=0.01$ ) and this study is the very recent study performed from 2015 to $2016 .^{7}$

Eradication rate of the first-line therapy was 90\% in the tailored group, which was consistent with previous studies. ${ }^{20,23} \mathrm{Al}-$ though the ultimate eradication rate did not differ between the two groups, the eradication rate of first-line therapy was 20\% lower in the empirical group compared to the tailored group. Thirty percent of patients in the empirical group received unnecessary antibiotics as a first-line therapy. To overcome high failure rate of empirical therapy, especially TT for 7 days, regimen of empirical therapy should be changed in Korea. However, BQT, sequential therapy or concomitant therapy did not show superiority over TT for the first-line eradication regimen in Korea. ${ }^{24,25}$ Further studies are anticipated in the future.

In Korea, TT for 7 days is a standard regimen as first-line therapy for $H$. pylori eradication therapy and reimbursed by National Health Insurance. Many guidelines recommend that longer duration of TT such as 14 days duration can achieve higher eradication rate. ${ }^{5}$ Two meta-analyses reported that 14day TT showed higher eradication rate compared with that of TT for 7 days. ${ }^{26,27}$ However, previous study in Korea showed that TT for 7 days was not inferior to TT for 14 days. ${ }^{28}$ In the current study, eradication rate of the first-line therapy and the ultimate eradication rate was $98.4 \%$ and $100 \%$, respectively in clarithromycin sensitive group. Considering that success rate of $H$. pylori culture is not so high, DPO-based PCR can be a good option to determine the regimen in areas with high clarithromycin resistance. In the tailored group, metronidazole was included both in the first-line and the second-line eradication for patients who were clarithromycin resistant and failed first-line eradication. Because this study is retrospective cohort study, eradication regimen was not standardized. Eradication regimen was chosen under physician's discretion. Metronidazole resistance can be overcome with prolonging treatment duration. ${ }^{29}$ For those patients, we extended eradication duration to 14 days. Among seven patients who received metronidazole twice, eradication was done in six patients (85.7\%).

Total costs were higher in the tailored group. However, due to higher eradication failure of the first-line therapy, cost for eradication regimen and ${ }^{13} \mathrm{C}$-UBT was higher in the empirical group. The cost of DPO-based PCR was 40 USD higher than the other diagnostic methods, which contributed to higher total medical cost in the tailored group.

Although we could not prove the cost-effectiveness of clarithromycin resistance test using DPO-based PCR, there are some points to expand this concept. First, the cost of eradication regimen, diagnostic methods, and ${ }^{13} \mathrm{C}$-UBT vary among the countries. Second, we did not include the cost for outpatient clinic registration. Patients in the empirical group visited outpatient clinic more because of higher first-line eradication failure. If we included cost for outpatient clinic to total cost, the difference of two groups might be decreased.

We developed an economic model to expect the point where DPO-based PCR would be economically justified under various eradication rate and treatment duration of empirical treatment. Because eradication regimen is chosen according to the clarithromycin resistance, there might be less likelihood of variability for successful eradication cost in the tailored group. Cost for one successful eradication will be increased when the first-line eradication or the ultimate eradication rate is low for empirical treatment. When eradication regimen is prescribed for 7 days, there would be no cost effectiveness in any eradication rate. However, tailored therapy using DPO-based PCR has costbenefit when the first-line eradication rate of 14-day empirical 
treatment was $80 \%$ or less. We also suggest that tailored treatment is cost-effective when the price of DPO-based PCR was cut down by 30 USD, given the current eradication rate of tailored treatment and empirical treatment.

With the increase of clarithromycin resistance, usefulness of DPO-based PCR was proved in several studies. ${ }^{19,20}$ However, cost-effectiveness should be justified to adopt this strategy as standard diagnostic method. Thus, our study has strength to investigate the cost-effectiveness of DPO-based PCR using economic modelling for the first time. Because there was no study regarding economic analysis of $H$. pylori eradication, we referred to economic modelling regarding prophylactic clipping for prevention of bleeding after post-polypectomy bleeding. ${ }^{21}$

There are several limitations in the current study. First, because of retrospective design in nature, some data were missing and selection bias might have occurred. History of $H$. pylori eradication which was important for clarithromycin resistance was available in $25.6 \%$ patients. Compliance and adverse events were not investigated. Second, baseline characteristics including indication for endoscopy differed between the two groups. Patients who received endoscopic resection for the treatment of early gastric neoplasia were $48.6 \%$ in the tailored group and $16.4 \%$ in the empirical group ( $<<0.01$ ). Because DPO-based PCR was done under physician's discretion, more patients who received endoscopic treatment underwent DPO-based PCR. However, patient's characteristics did not affect eradication of $H$. pylori. Third, metronidazole was included both in the first-line and the second-line eradication regimen in patients who showed clarithromycin resistance. Although metronidazole resistance can be overcome by extension of treatment duration, choice of eradication regimen was not proper. For second-line eradication who are resistant to clarithromycin, alternative eradication regimen such as fluoroquinolone replacing metronidazole might be proper. Further studies are required for these patients.

In conclusion, although the first-line eradication rate was higher in the tailored group compared to empirical group, the ultimate eradication rate did not differ between the two groups. We developed an economic modeling of $H$. pylori eradication. Based on our economic modeling, the cost of ultimate one successful eradication using DPO based PCR was lower than the cost of 14-day empirical treatment, especially under the circumstances with the first-line eradication rate cannot reach $80 \%$. This treatment strategy did not show cost-effectiveness compared to 7-day empirical treatment. This can be elucidated further with prospective studies.

\section{CONFLICTS OF INTEREST}

No potential conflict of interest relevant to this article was reported.

\section{REFERENCES}

1. Lee JW, Kim N, Kim JM, et al. Prevalence of primary and secondary antimicrobial resistance of Helicobacter pylori in Korea from 2003 through 2012. Helicobacter 2013;18:206-214.

2. Megraud F, Coenen S, Versporten A, et al. Helicobacter pylori resistance to antibiotics in Europe and its relationship to antibiotic consumption. Gut 2013;62:34-42.

3. Sasaki H, Nagahara A, Hojo M, et al. Ten-year trend of the cumulative Helicobacter pylori eradication rate for the 'Japanese eradication strategy'. Digestion 2013;88:272-278.

4. Shin WG, Lee SW, Baik GH, et al. Eradication rates of Helicobacter pylori in Korea over the past 10 years and correlation of the amount of antibiotics use: nationwide survey. Helicobacter 2016; 21:266-278.

5. Chey WD, Leontiadis GI, Howden CW, Moss SF. ACG clinical guideline: treatment of Helicobacter pylori onfection. Am J Gastroenterol 2017;112:212-239.

6. Horiki N, Omata F, Uemura M, et al. Annual change of primary resistance to clarithromycin among Helicobacter pylori isolates from 1996 through 2008 in Japan. Helicobacter 2009;14:86-90.

7. Hwang TJ, Kim N, Kim HB, et al. Change in antibiotic resistance of Helicobacter pylori strains and the effect of A2143G point mutation of $23 \mathrm{~S}$ rRNA on the eradication of $\mathrm{H}$. pylori in a single center of Korea. J Clin Gastroenterol 2010;44:536-543.

8. Graham DY, Lee SY. How to effectively use bismuth quadruple therapy: the good, the bad, and the ugly. Gastroenterol Clin North Am 2015;44:537-563.

9. Liou JM, Fang YJ, Chen CC, et al. Concomitant, bismuth quadruple, and 14-day triple therapy in the first-line treatment of Helicobacter pylori: a multicentre, open-label, randomised trial. Lancet 2016;388:2355-2365.

10. Liou JM, Bair MJ, Chen CC, et al. Levofloxacin sequential therapy vs levofloxacin triple therapy in the second-line treatment of Helicobacter pylori: a randomized trial. Am J Gastroenterol 2016;111: 381-387.

11. Chen Q, Zhang W, Fu Q, et al. Rescue therapy for Helicobacter pylori eradication: a randomized non-inferiority trial of amoxicillin or tetracycline in bismuth quadruple therapy. Am J Gastroenterol 2016;111:1736-1742.

12. Suzuki S, Gotoda T, Kusano C, Iwatsuka K, Moriyama M. The efficacy and tolerability of a triple therapy containing a potassiumcompetitive acid blocker compared with a 7-day PPI-based lowdose clarithromycin triple therapy. Am J Gastroenterol 2016;111: 949-956.

13. Park CS, Lee SM, Park CH, et al. Pretreatment antimicrobial susceptibility-guided vs. clarithromycin-based triple therapy for Helicobacter pylori eradication in a region with high rates of multiple drug resistance. Am J Gastroenterol 2014;109:1595-1602.

14. Cosme A, Lizasoan J, Montes M, et al. Antimicrobial susceptibility-guided therapy versus empirical concomitant therapy for eradication of Helicobacter pylori in a region with high rate of 
clarithromycin resistance. Helicobacter 2016;21:29-34.

15. Toracchio S, Cellini L, Di Campli E, et al. Role of antimicrobial susceptibility testing on efficacy of triple therapy in Helicobacter pylori eradication. Aliment Pharmacol Ther 2000;14:1639-1643.

16. Romano M, Iovene MR, Montella F, Vitale LM, De Simone T, Del Vecchio Blanco C. Pretreatment antimicrobial-susceptibility testing in the eradication of $\mathrm{H}$. pylori infection. Am J Gastroenterol 2000;95:3317-3318.

17. Bae SE, Jung HY, Kang J, et al. Effect of Helicobacter pylori eradication on metachronous recurrence after endoscopic resection of gastric neoplasm. Am J Gastroenterol 2014;109:60-67.

18. Woo HY, Park DI, Park H, et al. Dual-priming oligonucleotidebased multiplex PCR for the detection of Helicobacter pylori and determination of clarithromycin resistance with gastric biopsy specimens. Helicobacter 2009;14:22-28.

19. Lehours P, Siffré E, Mégraud F. DPO multiplex PCR as an alternative to culture and susceptibility testing to detect Helicobacter pylori and its resistance to clarithromycin. BMC Gastroenterol 2011; 11:112.

20. Lee HJ, Kim JI, Cheung DY, et al. Eradication of Helicobacter pylori according to $23 \mathrm{~S}$ ribosomal RNA point mutations associated with clarithromycin resistance. J Infect Dis 2013;208:1123-1130.

21. Bahin FF, Rasouli KN, Williams SJ, Lee EY, Bourke MJ. Prophylactic clipping for the prevention of bleeding following wide-field endoscopic mucosal resection of laterally spreading colorectal lesions: an economic modeling study. Endoscopy 2016;48:754-761.

22. Hu Y, Zhu Y, Lu NH. Primary antibiotic resistance of Helicobacter pylori in China. Dig Dis Sci 2017;62:1146-1154.
23. Liu Q, Qi D, Kang J, et al. Efficacy of real-time PCR-based detection of Helicobacter pylori infection and genotypic resistanceguided quadruple therapy as the first-line treatment for functional dyspepsia with Helicobacter pylori infection. Eur J Gastroenterol Hepatol 2015;27:221-225.

24. Jang HJ, Choi MH, Kim YS, et al. Effectiveness of triple therapy and quadruple therapy for Helicobacter pylori eradication. Korean J Gastroenterol 2005;46:368-372.

25. Jung YS, Park CH, Park JH, Nam E, Lee HL. Efficacy of Helicobacter pylori eradication therapies in Korea: a systematic review and network meta-analysis. Helicobacter 2017;22:e12389.

26. Yuan Y, Ford AC, Khan KJ, et al. Optimum duration of regimens for Helicobacter pylori eradication. Cochrane Database Syst Rev 2013;(12):CD008337.

27. Calvet X, García N, López T, Gisbert JP, Gené E, Roque M. A meta-analysis of short versus long therapy with a proton pump inhibitor, clarithromycin and either metronidazole or amoxycillin for treating Helicobacter pylori infection. Aliment Pharmacol Ther 2000;14:603-609.

28. Kim BG, Lee DH, Ye BD, et al. Comparison of 7-day and 14-day proton pump inhibitor-containing triple therapy for Helicobacter pylori eradication: neither treatment duration provides acceptable eradication rate in Korea. Helicobacter 2007;12:31-35.

29. Filipec Kanizaj T, Katicic M, Skurla B, Ticak M, Plecko V, Kalenic S. Helicobacter pylori eradication therapy success regarding different treatment period based on clarithromycin or metronidazole tripletherapy regimens. Helicobacter 2009;14:29-35. 